\title{
Comparative study of a new commercial recombinant line assay and two immunoassays for detection of SARS-CoV-2 IgG antibodies in PCR-confirmed outpatients
}

https://doi.org/10.1515/labmed-2020-0095

Received August 17, 2020; accepted November 8, 2020;

published online December 14, 2020

\section{Abstract}

Objectives: Commercially available immunoassays have been developed for detection of antibodies against SARS-CoV-2. However, equivocal and discrepant results between different immunoassay can occur requiring further assessment by confirmatory tests.

Methods: We investigated the new commercial line assay recomLine SARS-CoV-2 IgG (Mikrogen, containing the antigens S1, receptor-binding domain of the spike protein, and nucleocapsid protein (NP) of SARS-CoV-2) within a collection of well characterized serum samples from COVID-19 outpatients ( $\mathrm{n}=49)$ and SARS-CoV-2-PCR-positive asymptomatic contact persons $(n=6)$ in comparison to two commercial immunoassays, the S1 antigen based Anti-SARS-CoV-2-ELISA IgG by Euroimmun and the NP based Elecsys ${ }^{\circledR}$ Anti-SARS-CoV-2 by Roche.

Results: The recomLine assay was positive in all samples which had an equivocal or positive result for SARS-CoV-2 antibodies in at least one of the two immunoassays. It showed high agreement with the overall results of the immunoassays $(94.5 \%$ [Cohen's kappa $=0.85$ ] and 92.7\% [Cohen's kappa 0.81 to the ELISA by Euroimmun and the assay by Roche, respectively). In addition, high agreement of the reactivity to the specific antigens S1 and NP in the recomLine assays compared to the results of the S1 based ELISA and NP based Elecsys ${ }^{\circledR}$ assay, was found (90.9\% [Cohen's kappa 0.78] and 96.4\% [Cohen's kappa 0.91] for S1 and NP, respectively).

Conclusions: The new recomLine SARS-CoV-2 IgG assay may be used as an additional tool for investigation of

\footnotetext{
*Corresponding author: Nele Wellinghausen, MVZ Labor Ravensburg, Elisabethenstr. 11, 88212 Ravensburg, Germany,

E-mail: nele.wellinghausen@labor-gaertner.de

Ralitsa Ivanova, Susanne Deininger and Andrea Götz, MVZ Labor

Ravensburg, Ravensburg, Germany
}

equivocal or discrepant results of Anti-SARS-CoV-2 immunoassays and for antigen-specific detection of SARS-CoV-2 IgG antibodies.

Keywords: immunoblot; immunoassay; line assay; SARS-CoV-2; serology.

\section{Introduction}

SARS-CoV-2 is a new coronavirus which causes an acute respiratory disease named COVID-19. It has led to a worldwide pandemic declared by the World Health Organization (WHO) on March 11th 2020. While diagnosis of acute infection with SARS-CoV-2 is done by RT-PCR in respiratory samples, serological testing, especially determination of SARS-CoV-2 IgG antibodies, has been implemented for epidemiological studies and assessment of infection status in individuals. A variety of diagnostic tests including highthroughput immunoassays on random access platforms are commercially available by now. These tests differ with respect to assay principle, test format, and SARS-CoV-2 antigens used as well as diagnostic sensitivity and specificity [1-6]. Therefore, divergent results in different serological assays and discrepant results compared to SARS-CoV-2-RT-PCR do occur in some patients. In addition, equivocal or borderline and weak positive results in some SARS-CoV-2 IgG assays in individual patients do not allow definite interpretation of SARS-CoV-2 serology.

Recently, a new recombinant line assay for detection of SARS-CoV-2 IgG has been brought to market (recomLine SARS-CoV-2 IgG, Mikrogen). It allows detection of IgG antibodies against three antigens of SARS-CoV-2, i.e. the S1 protein, the receptor-binding domain (RBD) of the spike protein, and the nucleocapsid protein (NP). It may, thus, be a valuable tool for investigation of unclear or divergent results of other serological assays.

In this first comparative study, we tested the new recomLine SARS-CoV-2 IgG assay with a collection of well characterized serum samples from COVID-19 outpatients 
and asymptomatic contact persons and compared the results to two commercial immunoassays, one detecting SARS-CoV-2 IgG against the S1 antigen (Anti-SARS-CoV2-ELISA IgG by Euroimmun) and one detecting SARS-CoV-2 antibodies against the NP (Elecsys ${ }^{\circledR}$ Anti-SARS-CoV-2 by Roche).

\section{Materials and methods}

\section{Immunoassays for SARS-CoV-2 antibody testing}

The Anti-SARS-CoV-2-ELISA IgG (Euroimmun, Luebeck, Germany, recombinant S1 protein based, expressed in cell line HEK 293) was performed fully automated on the Euroimmun Workstation ELISA according to the manufacturer's instructions. Samples with a ratio $<0.8$ are interpreted as negative, $0.8-1.09$ as equivocal, and $\geq 1.1$ as positive. Elecsys ${ }^{\circledR}$ Anti-SARS-CoV-2 (recombinant NP antigen based, detection of total antibodies against SARS-CoV-2, no differentiation of IgM, IgA and IgG) was run on the cobas ${ }^{\mathrm{TM}} \mathrm{e} 801$ (Roche Diagnostics, Mannheim, Germany). Samples with a cutoff index (COI) $<1.0$ are interpreted as negative and $\geq 1.0$ as positive. The recomLine SARS-CoV2 IgG line immunoassay (Mikrogen, Neuried, Germany), lot LCS062003 for RUO representing the version submitted for CE validation by the manufacturer, was performed on the automated Dynablot blot processing system (Dynex, Praha, Czeck Republic). The recomLine IgG strip contains the following recombinant antigens: NP, RBD, and spike protein S1 of SARS-CoV-2, and NP of hCoV 229E, NL63, OC43, and HKU1. Intensity of the detected antigen bands were determined by automated reading in the Microscan system (Mikrogen) and classified by the software from + to +++ . According to the manufacturer detection of at least one antigen out of NP, RBD, and S1 by SARS-CoV-2 represents a positive IgG result. In each run a positive control serum from a PCR-confirmed COVID-19 patient and a negative control from a patient without SARS-CoV-2 infection were included.

\section{Serum samples and patient characteristics}

Serum samples were sent to our laboratory from ambulatory patients for determination of SARS-CoV-2 IgG. The serum collection used for validation of the line assay included 49 samples from 41 outpatients with past, ambulatory treated COVID-19 infection confirmed by SARS-CoV-2-RT-PCR in nasopharyngeal swabs and six samples from five asymptomatic contact persons with PCR-confirmed SARS-CoV-2 infection. From three patients four or three samples and from one asymptomatic contact person two serum samples were available. Samples were stored at $4-8{ }^{\circ} \mathrm{C}$ for up to one week, then frozen at $-20^{\circ} \mathrm{C}$, and thawed only once.

SARS-CoV-2-RT-PCR was performed with different commercial PCR assays on the cobas ${ }^{\circledR} 6800$ analyzer and the LightCycler ${ }^{\circledR}$ 480II (Roche Diagnostics) targeting the envelope (E) gene, open reading frame (orf) 1, and/or the nucleocapsid gene as described previously [7]. Generally, two of the above genes were amplified. However, in 14 patients (no. 1, 7, 9, 15, 22, 24-28, 31, 34, 41, and 43) amplification of a second SARS-CoV-2-specific gene apart from the $\mathrm{E}$ gene was not done according to the former recommendations for SARS-CoV-2 detection in high incidence settings during March and April 2020.

\section{Statistical analyses}

Assessment of agreement was performed by Cohen's kappa using the software tool Analyse-it for Micorsoft Excel 4.65.2 (Leeds, UK). In addition to Cohen's kappa the 95\% confidence interval (CI) and standard error (SE) were calculated.

\section{Results}

Out of the 38 PCR-confirmed COVID-19 patients with only one serum sample investigated, 29 revealed concordant results in both immunoassays ( $\mathrm{n}=21$ positive, $\mathrm{n}=8$ negative). In all samples the respective results were confirmed by the recomLine assay (Table 1). Even weak positive results (ratio 1.1 to 1.5) in the S1-antigen-based AntiSARS-CoV-2 IgG ELISA by Euroimmun (samples 2, 3, and 10) or the Anti-SARS-CoV-2 assay by Roche (COI 1.0-1.4, samples 7 and 21) were positive in the recomLine assay for IgG antibodies against the S1 or NP antigen, respectively (Table 1). Samples from three asymptomatic contact persons with negative results in both immunoassays (samples 42-44) were also confirmed as negative by the recomLine assay. Regarding patients with divergent results between the two immunoassays or equivocal result in the ELISA (ratio 0.8-1.09) ( $\mathrm{n}=10$, samples $30-38$ and 45), all were confirmed positive by the recomLine assay. Agreement between the results of the recomLine assay and the ELISA by Euroimmun and the assay by Roche were 94.5\% (kappa=0.85, CI 0.69-1.0, SE 0.082) and 92.7\% (kappa 0.81, CI 0.63-0.99, SE 0.01).

Regarding reactivity to the respective SARS-CoV-2 antigen, samples with a negative or equivocal result in the S1 antigen based Anti-SARS-CoV-2 IgG ELISA by Euroimmun but a positive result in the NP based Anti-SARS-CoV-2 assay by Roche were positive for IgG against $\mathrm{S} 1$ antigen in the recomLine assay in five out of seven samples (samples 30-35 and 38). The two samples which were negative in the Anti-SARS-CoV-2 assay by Roche but positive in the AntiSARS-CoV-2 IgG ELISA by Euroimmun (samples 36 and 37) were both confirmed positive for the S1 antigen by the recomLine assay and in one sample even IgG against NP was detected by the recomLine assay in high intensity. Agreement between the results of the S1 antigen based ELISA and reactivity against $\mathrm{S} 1$ in the recomLine assay was 90.9\% (kappa $=0.78$, CI 0.59-0.96, SE 0.78), and between the results of the NP based assay and reactivity against NP in the recomLine assay was $96.4 \%$ (kappa $=0.91$, CI 0.78-1.00, SE 0.064).

Follow-up samples were available from three ambulatory treated COVID-19 patients and one asymptomatic 
Table 1: Results of immunoassays and SARS-CoV-2 IgG recomLine assay in PCR-confirmed COVID-19 patients and asymptomatic contact persons.

\begin{tabular}{|c|c|c|c|c|c|c|c|c|c|}
\hline \multirow[b]{2}{*}{ No. } & \multicolumn{2}{|c|}{$\begin{array}{r}\text { Anti-SARS-CoV-2 ELISA IgG } \\
\text { (Euroimmun) (S1 antigen) }\end{array}$} & \multicolumn{2}{|c|}{$\begin{array}{r}\text { Elecsys } ® \text { Anti-SARS-CoV-2 } \\
\text { (Roche) (NP antigen) }\end{array}$} & \multicolumn{4}{|c|}{ recomLine SARS-CoV-2 IgG (Mikrogen) } & \multirow[b]{2}{*}{ Day } \\
\hline & Ratio $^{a}$ & Qual & $\mathrm{COI}^{\mathrm{b}}$ & Qual & S1 & RBD & NP & Qual & \\
\hline 1 & 12.3 & pos & 74.4 & pos & +++ & +++ & +++ & pos & 30 \\
\hline 2 & 1.15 & pos & 35.3 & pos & + & + & +++ & pos & 50 \\
\hline 3 & 1.15 & pos & 6.99 & pos & + & + & ++ & pos & 10 \\
\hline 4 & 8.6 & pos & 14.4 & pos & +++ & +++ & +++ & pos & 24 \\
\hline 5 & 1.98 & pos & 7.55 & pos & ++ & + & +++ & pos & 25 \\
\hline 6 & 1.59 & pos & 3.02 & pos & ++ & + & ++ & pos & 26 \\
\hline 7 & 1.91 & pos & 1.33 & pos & ++ & ++ & ++ & pos & 31 \\
\hline 8 & 2.43 & pos & 39.9 & pos & ++ & ++ & +++ & pos & 17 \\
\hline 9 & 2.20 & pos & 26.3 & pos & + & + & +++ & pos & 27 \\
\hline 10 & 1.27 & pos & 6.99 & pos & + & + & +++ & pos & 28 \\
\hline 11 & 2.00 & pos & 6.88 & pos & + & + & + & pos & 20 \\
\hline 12 & 1.97 & pos & 3.91 & pos & ++ & + & ++ & pos & 28 \\
\hline 13 & 19.3 & pos & 64.9 & pos & +++ & +++ & +++ & pos & 33 \\
\hline 14 & 8.33 & pos & 41.8 & pos & +++ & +++ & +++ & pos & 33 \\
\hline 15 & 5.91 & pos & 2.63 & pos & +++ & +++ & +++ & pos & 21 \\
\hline 16 & 4.58 & pos & 2.58 & pos & +++ & ++ & + & pos & 23 \\
\hline 17 & 6.50 & pos & 18.3 & pos & +++ & +++ & +++ & pos & 23 \\
\hline 18 & 2.20 & pos & 11.0 & pos & + & + & +++ & pos & 12 \\
\hline 19 & 6.57 & pos & 75.3 & pos & +++ & +++ & +++ & pos & 32 \\
\hline 20 & 14.7 & pos & 72.0 & pos & +++ & +++ & +++ & pos & 23 \\
\hline 21 & 1.61 & pos & 1.24 & pos & + & - & + & pos & 35 \\
\hline 22 & 0.29 & neg & 0.38 & neg & - & - & - & neg & 22 \\
\hline 23 & 0.57 & neg & 0.09 & neg & - & - & - & neg & 41 \\
\hline 24 & 0.04 & neg & 0.08 & neg & - & - & - & neg & 28 \\
\hline 25 & 0.19 & neg & 0.07 & neg & - & - & - & neg & 68 \\
\hline 26 & 0.25 & neg & 0.07 & neg & - & - & - & neg & 54 \\
\hline 27 & 0.24 & neg & 0.07 & neg & - & - & - & neg & 40 \\
\hline 28 & 0.54 & neg & 0.38 & neg & - & - & - & neg & 25 \\
\hline 29 & 0.23 & neg & 0.07 & neg & - & - & - & neg & 36 \\
\hline 30 & 0.94 & equi & 2.09 & pos & - & - & + & pos & 56 \\
\hline 31 & 1.06 & equi & 2.19 & pos & - & - & ++ & pos & 64 \\
\hline 32 & 1.06 & equi & 14.6 & pos & ++ & + & ++ & pos & 23 \\
\hline 33 & 0.85 & equi & 9.05 & pos & + & - & +++ & pos & 18 \\
\hline 34 & 0.92 & equi & 22.6 & pos & ++ & - & + & pos & 84 \\
\hline 35 & 0.82 & equi & 9.28 & pos & + & - & ++ & pos & 66 \\
\hline 36 & 4.05 & pos & 0.67 & neg & +++ & +++ & +++ & pos & 32 \\
\hline 37 & 3.72 & pos & 0.16 & neg & +++ & +++ & - & pos & 15 \\
\hline 38 & 0.27 & neg & 11.9 & pos & + & + & +++ & pos & 19 \\
\hline $42^{d}$ & 0.11 & neg & 0.08 & neg & - & - & - & neg & 14 \\
\hline $43^{d}$ & 0.19 & neg & 0.06 & neg & - & - & - & neg & 13 \\
\hline $44^{d}$ & 0.25 & neg & 0.06 & neg & - & - & - & neg & 56 \\
\hline $45^{d}$ & 2.31 & pos & 0.32 & neg & +++ & +++ & - & pos & 44 \\
\hline
\end{tabular}

${ }^{\mathrm{a}}$ Ratio $<0.8$ negative (neg), $0.8-1.09$ equivoal (equi), $\geq 1.1$ positive (pos). ${ }^{\mathrm{b}}$ Cut-off index (COI) $<1.0$ negative, $\geq 1.0$ positive. ${ }^{\mathrm{C}}$ Day after onset of symptoms or day after the PCR-positive nasopharyngeal swab in asymptomatic contact persons. ${ }^{\mathrm{d}}$ Asymptomatic PCR-positive contact person.

PCR-confirmed contact person. Reactivity of SARS-CoV-2 IgG against $\mathrm{S} 1$ and NP correlated well with the results of immunoassays (Table 2). In sample 40 only, the immunoassays detected SARS-CoV-2 antibodies at an earlier time point than the recomLine assay. Intensity of the IgG bands against the NP was more pronounced at an earlier time point after infection in both the Elecsys ${ }^{\circledR}$ Anti-SARS-CoV-2 and the recomLine assay.

In addition to the SARS-CoV-2 antibodies, IgG antibodies against NP of one or more seasonal hCoV were detected 37 of the 46 patients ( $80.4 \%$, data not shown) by the recomLine assay. 
Table 2: Results of immunoassays and recomLine assay in PCR-confirmed COVID-19 patients with follow-up serum samples.

\begin{tabular}{|c|c|c|c|c|c|c|c|c|c|}
\hline \multirow[b]{2}{*}{ No. } & \multicolumn{2}{|c|}{$\begin{array}{r}\text { Anti-SARS-CoV-2-ELISA IgG } \\
\text { (Euroimmun) (S1 antigen) }\end{array}$} & \multicolumn{2}{|c|}{$\begin{array}{r}\text { Elecsys } ® \text { Anti-SARS-CoV-2 } \\
\text { (Roche) (NP antigen) }\end{array}$} & \multicolumn{4}{|c|}{ recomLine SARS-CoV-2 IgG (Mikrogen) } & \multirow[b]{2}{*}{ Dayc $^{c}$} \\
\hline & Ratio $^{a}$ & qual & $\mathrm{COI}^{\mathrm{b}}$ & qual & S1 & RBD & NP & qual & \\
\hline 39a & 0.69 & neg & 0.23 & neg & - & - & - & neg & 20 \\
\hline $39 b$ & 0.86 & equi & 3.05 & pos & + & + & +++ & pos & 27 \\
\hline $39 c$ & 2.18 & pos & 6.33 & pos & ++ & ++ & +++ & pos & 40 \\
\hline $39 d$ & 1.24 & pos & 10.1 & pos & ++ & ++ & +++ & pos & 68 \\
\hline $40 a$ & 1.07 & equi & 2.67 & pos & - & - & - & neg & 18 \\
\hline $40 \mathrm{~b}$ & 1.37 & pos & 1.71 & pos & - & - & + & pos & 25 \\
\hline $40 c$ & 1.75 & pos & 10.5 & pos & + & + & + & pos & 39 \\
\hline $40 \mathrm{~d}$ & 1.07 & equi & 7.14 & pos & + & + & + & pos & 67 \\
\hline $41 a$ & 1.8 & pos & 10.7 & pos & + & + & +++ & pos & 19 \\
\hline $41 b$ & 2.22 & pos & 10 & pos & ++ & ++ & +++ & pos & 31 \\
\hline $41 c$ & 1.51 & pos & 24.8 & pos & + & + & +++ & pos & 81 \\
\hline $46 a^{d}$ & 0.47 & neg & 2.01 & pos & - & - & ++ & pos & 14 \\
\hline $46 b^{d}$ & 1.76 & pos & 67.1 & pos & ++ & ++ & +++ & pos & 38 \\
\hline
\end{tabular}

${ }^{a}$ Ratio $<0.8$ negative (neg), $0.8-1.09$ equivoal (equi), $\geq 1.1$ positive (pos). ${ }^{b}$ Cut-off index (COI) $<1.0$ negative, $\geq 1.0$ positive. ${ }^{c}$ Day after onset

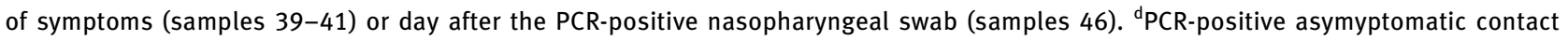
persons.

\section{Discussion}

Determination of SARS-CoV-2 IgG antibodies for epidemiological reasons and diagnosis of past infection with SARS-CoV-2 has already found its way into routine diagnostics as the SARS-CoV-2 pandemia has evolved further. For this purpose, a variety of commercial immunoassays are available by now. Although immunoassays cannot determine the neutralizing ability of SARS-CoV-2 IgG they facilitate evaluation of seroprevalence and results of some tests have been shown to correlate positively with the results of neutralization tests (NT) $[2,3,8]$. Interpretation of SARS-CoV-2 serology is difficult in some patients since equivocal or weak positive results may represent false positive results, and discrepant results between different immunoassays do occur. We, therefore, investigated the new commercial SARS-CoV-2 IgG recomLine assay in comparison to two commercial immunoassays for detection of SARS-CoV-2 IgG in patients with past SARS-CoV-2 infection and asymptomatic contact persons. A prototype of this test was already investigated in a small number of patients before and showed promising results compared to several commercial immunoassays [9]. However, this pre-market version of the recomLine assay did only detect IgG antibodies against NP of SARS-CoV-2 and not against $\mathrm{S} 1$ and RBD.

In our study, the recomLine assay showed excellent agreement with the results of two immunoassays, the AntiSARS-CoV-2 ELISA IgG by Euroimmun and the Anti-
SARS-CoV-2 total antibody assay by Roche. We used the immunoassays by Euroimmun and Roche for comparison since these tests are used in our routine workup in the laboratory and since they use different antigens of SARS-CoV-2, i.e. the S1 antigen by Euroimmun and the NP by Roche. Besides positive and negative samples, also samples with equivocal or weak positive results in one of the immunoassays were detected positive in the recomLine assay. In addition, the antigen specificity of the SARS-CoV-2 IgG detected in the recomLine assay, i.e. against $\mathrm{S} 1 \mathrm{and} /$ or $\mathrm{NP}$, also showed high agreement to the results of the immunoassays.

Discrepant results between the results of the recomLine assay and the immunoassays were observed in four samples only. Discrepant results may be caused by different reasons: First, the nature of antigen used by the manufacturers and the epitopes detected by the antibodies are certainly different due to different expression systems and production steps. Unfortunately, the manufacturers do not present detailed information regarding the nature and production of their test antigens. Second, the dynamics of antibody response to different SARS-CoV-2 antigen epitopes differs between individuals and may be reflected by differing results of the assays. Thus, we confirm earlier data $[3,8,10]$ regarding an inter-individual variation of antibody response against SARS-CoV-2 antigens with exclusive antibody response against S1 protein or NP in some patients. Third, while the Anti-SARS-CoV-2 ELISA by Euroimmun and the recomLine assay detect IgG antibodies 
only, the Anti-SARS-CoV-2 assay by Roche also detects IgA and IgM antibodies, to an albeit lesser extent than IgG. The isotype and also the avidity of antibodies may also influence the assay result. Lastly, the severity of disease influences antibody response in SARS-CoV-2 infection. It has been shown in several studies that SARS-CoV-2 antibody response appears earlier and is more pronounced in severely ill patients compared to less severe cases [11, 12]. In addition, the kinetics of antibody decline may differ as recent studies suggest individual variation in antibody decline [13]. These factors may influence the test results of different serological assays and could not be systematically considered in our study since the severity of disease in the COVID-19 patients was not known apart from the fact that all patients were treated as outpatients.

Antibodies against the four hCoV 229E, NL63, OC43, and HKU1 were found in $80 \%$ of the patients by recomLine assay in our study and confirmed others like the manufacturer stating a high rate of seropositivity for seasonal $\mathrm{hCoV}$ in blood donors (package insert of the recomLine assay) and also in COVID-19 patients [14]. The manufacturer of the recomLine assay did not find cross-reactivity of the recomLine assay with seasonal hCoV but samples from only nine patients with $\mathrm{hCoV}$ infection were included. Investigation of possible serological cross-reactivity between SARS-CoV-2 and hCoV were not aim of the present study but may be investigated in further studies.

Although we investigated serum samples from well characterized, PCR-confirmed patients with past COVID-19 and asymptomatic contact persons, our study as some limitations: We were not able to perform neutralization assays for antibody detection and, thus, the accepted gold standard for SARS-CoV-2 serology is missing. Therefore, evaluation of sensitivity of the recomLine assays is not possible from our data. In addition, we investigated sera from patients with an equivocal or positive result in a SARS-CoV-2 immunoassay only but no serologically negative patients. Thus, specificity of the recomLine assay could not be determined in our study either. Nevertheless, we, for the first time, present results of the new commercial recomLine assay in comparison to well established immunoassays. As a supplement to the determination of specificity by the manufacturer, we, however, tested four sera from patients with primary Epstein-Barr-Virus infection and three sera from patients with highly positive rheumatoid factor and all samples were clearly negative in all three assays (data not shown). From a clinical point of view, it has to be mentioned that the detection of SARS-CoV-2 antibodies by either immunoassay allows determination of seropositivity and possible past infection but do not allow to determine immunity against COVID-19.

\section{Conclusions}

This first comparative investigation of the new commercial recomLine SARS-CoV-2 IgG line assay showed an excellent agreement between the overall results of the recomLine assay and two commercial immunoassays and also with respect to reactivity to the specific SARS-CoV-2 antigens S1 and NP, respectively. The recomLine assay may, thus, be used as an additional tool for investigation of equivocal or discrepant results of Anti-SARS-CoV-2 immunoassays and for antigen-specific detection of SARS-CoV-2 IgG antibodies.

Research funding: The recomLine SARS-CoV-2 IgG kits were supplied at no charge by Mikrogen.

Author contributions: All authors have accepted responsibility for the entire content of this manuscript and approved its submission.

Competing interests: Authors state no conflict of interest. Informed consent: Informed consent was obtained from all individuals included in this study.

Ethical approval: Research involving human subjects complied with all relevant national regulations, institutional policies and is in accordance with the tenets of the Helsinki Declaration (as revised in 2013). No further ethical approval was necessary for this diagnostic study.

\section{References}

1. Egger M, Bundschuh C, Wiesinger K, Gabriel C, Clodi M, Mueller T, et al. Comparison of the Elecsys ${ }^{\circledR}$ Anti-SARS-CoV-2 immunoassay with the EDI enzyme linked immunosorbent assays for the detection of SARS-CoV-2 antibodies in human plasma. Clin Chim Acta 2020;509:18-21.

2. Jääskeläinen AJ, Kuivanen $S$, Kekäläomem $S$, Ahava $M J$, Liinov R, Kallio-Kokko H, et al. Performance of six SARS-CoV-2 immunoassays in comparison with microneutralisation. J Clin Virol 2020;129:104512.

3. Kohmer N, Westhaus S, Ruehl C, Ciesek S, Rabenau HF. Brief clinical evaluation of six high-throughput SARS-CoV-2 IgG antibody assays. J Clin Virol 2020;129:104480.

4. Lassaunière R, Frische $A$, Harboe ZB, Nielsen ACY, Fomsgaard $A$, Krogfelt KA, et al. Evaluation of nine commercial SARS-CoV-2 immunoassays. medRxiv 2020. https://doi.org/10.1101/2020.04. 09.20056325.

5. Lippi G, Salvagno GL, Pegoraro M, Militello V, Caloi C, Peretti A, et al. Assessment of immune response to SARS-CoV-2 with fully automated MAGLUMI 2019-nCoV IgG and IgM chemiluminescence immunoassays. Clin Chem Lab Med 2020;58:1156-9.

6. Whitman JD, Hiatt J, Mowery CT, Shy BR, Yu R, Yamamoto TN, et al. Test performance evaluation of SARS-CoV-2 serological assays. medRxiv 2020. doi:https://doi.org/10.1101/2020.04.25.20074856.

7. Wellinghausen N, Plonne D, Voss M, Ivanova R, Frodl R, Deininger S. SARS-CoV-2-IgG response is different in COVID-19 outpatients and asymptomatic contact persons. J Clin Virol 2020;130:104542. 
8. Rijkers G, Murk JL, Wintermans B, van LB, van den Berge $M$, Veenemans J, et al. Differences in antibody kinetics and functionality between severe and mild severe acute respiratory syndrome coronavirus 2 infections. J Infect Dis 2020;222: 1265-9.

9. Stroemer A, Grobe O, Rose R, Fickenscher H, Lorentz T, Krumholz A. Diagnostic accuracy of six commercial SARS-CoV-2 IgG/total antibody assays and identification of SARS-CoV-2 neutralzing antibodies in convalescent sera. medRxiv 2020. https://doi.org/ 10.1101/2020.06.15.20131672.

10. Wellinghausen N, Voss M, Ivanova R, Deininger S. Evaluation of the SARS-CoV-2-IgG response in outpatients by five commercial immunoassays. GMS Infect Dis 2020;8:Doc22 [Epub ahead of print].
11. Kellam P, Barclay $W$. The dynamics of humoral immune responses following SARS-CoV-2 infection and the potential for reinfection. J Gen Virol 2020;101:791-7.

12. Zhao J, Yuan Q, Wang H, Liu W, Liao X, Su Y, et al. Antibody responses to SARS-CoV-2 in patients of novel coronavirus disease 2019. Clin Infect Dis 2020;71:2027-34.

13. Zhou W, Xu X, Chang Z, Wang H, Zhong X, Tong X, et al. The dynamic changes of serum IgM and IgG against SARS-CoV-2 in patients with COVID-19. J Med Virol 2020. https://doi.org/10. 1002/jmv.26353 [Epub ahead of print].

14. van Tol S, Mögling R, Li W, Godeke GJ, Swart A, Bergmans B, et al. Accurate serology for SARS-CoV-2 and common human coronaviruses using a multiplex approach. Emerg Microb Infect 2020;9:1965-73. 